\title{
Sur les peuplements de Genévrier thurifère (Juniperus thurifera L.) en Haut Pallars (Pyrénées centrales, Catalogne). Présentation géobotanique et écologique
}

\author{
Sobre las comunidades de sabina albar (Juniperus thurifera L.) \\ en el Alto Pallars (Pirineo Central catalán). \\ Presentación geobotánica y ecológica
}

Pere AYMERICH ${ }^{1} \&$ Luis VILLAR ${ }^{2}$

1. C/Barcelona, 29, E-08600 Berga (Barcelona)

E-mail : pere_aymerich@yahoo.es

2. Instituto Pirenaico de Ecología, IPE-CSIC. Apdo. 64, E-22700 Jaca (Huesca)

E-mail : Ivillar@ipe.csic.es

\begin{abstract}
Résumé
Le Genévrier thurifère (Juniperus thurifera L.) était connu depuis longtemps en Haute-Garonne et en Ariège et plus récemment dans les préPyrénées (Aragon) (quelques pieds). De nouvelles populations ont été découvertes en 2006 par l'un de nous (Aymerich 2007) en Haut Pallars, Lleida (Espagne). La plus importante population est celle du Vall Ferrera mais il y en existe d'autres, mineures, dans le Vall de Cardòs et même dans la vallée principale de la Noguera Pallaresa. Leur surface est estimée à 356 ha, représentant 37 quadrillages UTM de $1 \mathrm{~km}$ de côté. Le nombre d'individus est de l'ordre de 5000.
\end{abstract}

Ces bosquets colonisent les pentes pierreuses, assez raides et très ensoleillées de l'étage montagnard, entre (860) 1100-1450 (1600) mètres d'altitude, normalement sur des schistes pas trop acides. Ils jouissent d'un topoclimat relativement continental qui caractérise cette partie des Pyrénées. Parfois en compagnie du Genévrier commun (J. communis L.), I'arbre s'associe également au Genévrier de Phénicie (J. phoenicea L.), avec lequel il avait été confondu. Si certains de ces peuplements peuvent être considérés comme primaires, d'autres seraient secondaires, établis spontanément sur d'anciens pâturages ou champs abandonnés.

Nous présentons ici une esquisse phytotopographique, floristique et biogéographique de ces formations. Encore une fois, ces peuplements

Mots clés : J. thurifera, Haut Pallars, Pyrénées, phytotopographie, écologie. représenteraient les fragments isolés d'une aire de répartition plus continue dans la vallée moyenne de l'Èbre. Sans doute dans ces enclaves ont-elles pu résister à la concurrence des forêts de Quercus ilex L. subsp. ballota (Desf.) Samp. De même, l'impact des incendies, des parasites, l'action directe des herbivores, l'élagage ou d'autres exploitations humaines n'ont pu conduire à leur élimination. Aujourd'hui, les bosquets récupèrent et leur protection est assurée par le Parc naturel de l'Alt Pirineu, s'étendant entre le val d'Aran et l'Andorre.

\section{Resumen}

La sabina albar (Juniperus thurifera L.) se conocía hace tiempo de Haute-Garonne o Ariège (Francia) y recientemente-algunos pies-del Prepirineo Aragonés. Nuevas poblaciones fueron halladas en 2006 por uno de nosotros en el Alt Pallars, Lleida (España). Junto al núcleo principal de la Vall Ferrera hay otros secundarios en la Vall de Cardòs y en el propio río Noguera Pallaresa. Su área se estima en 356 ha, las cuales se reparten por 37 cuadrículas UTM de $1 \mathrm{~km}$ de lado. Su número de ejemplares se acerca a 5000.

Ocupan laderas pendientes, pedregosas y soleadas en el piso montano, entre (860) 1100-1450 (1600) $m$ de altitud, sobre esquistos poco o nada pobres en bases, bajo un clima relativamente continental de esta parte de los Pirineos. Suele 
convivir con enebros -J. communis L.- y alguna vez con sabina negral (J. phoenicea L.), con la que de lejos se había confundido. Junto a las poblaciones que podemos considerar primarias, observamos también otras secundarias, consecuencia de la colonización reciente de antiguos pastos e incluso campos abandonados.

En esta comunicación haremos un análisis fitotopográfico, florístico y biogeográfico de estos sabinares. En una primera interpretación representarían el resto de un área menos discontinuo por el valle medio del Ebro, gracias al hecho de que en esos enclaves han podido resistir la competencia de los bosques circundantes de Quercus ilex L. subsp. ballota (Desf.) Samp. Asimismo, los incendios, las plagas, el pastoreo y demás actividades humanas no han llegado a destruirlas. Ahora se recuperan y la gran mayoría gozan de protección legal, dentro del "Parc natural de I'Alt Pirineu », situado entre el valle de Arán y Andorra.

\section{Introduction}

La présence très isolée du Genévrier thurifère dans les Pyrénées centrales françaises est connue depuis le début du $\mathrm{XX}^{\mathrm{e}}$ siècle, époque où il a été découvert à la montagne de Rié, près de Marignac, en Haute-Garonne (Coste \& Soulié 1913). Quatre-vingts ans plus tard,

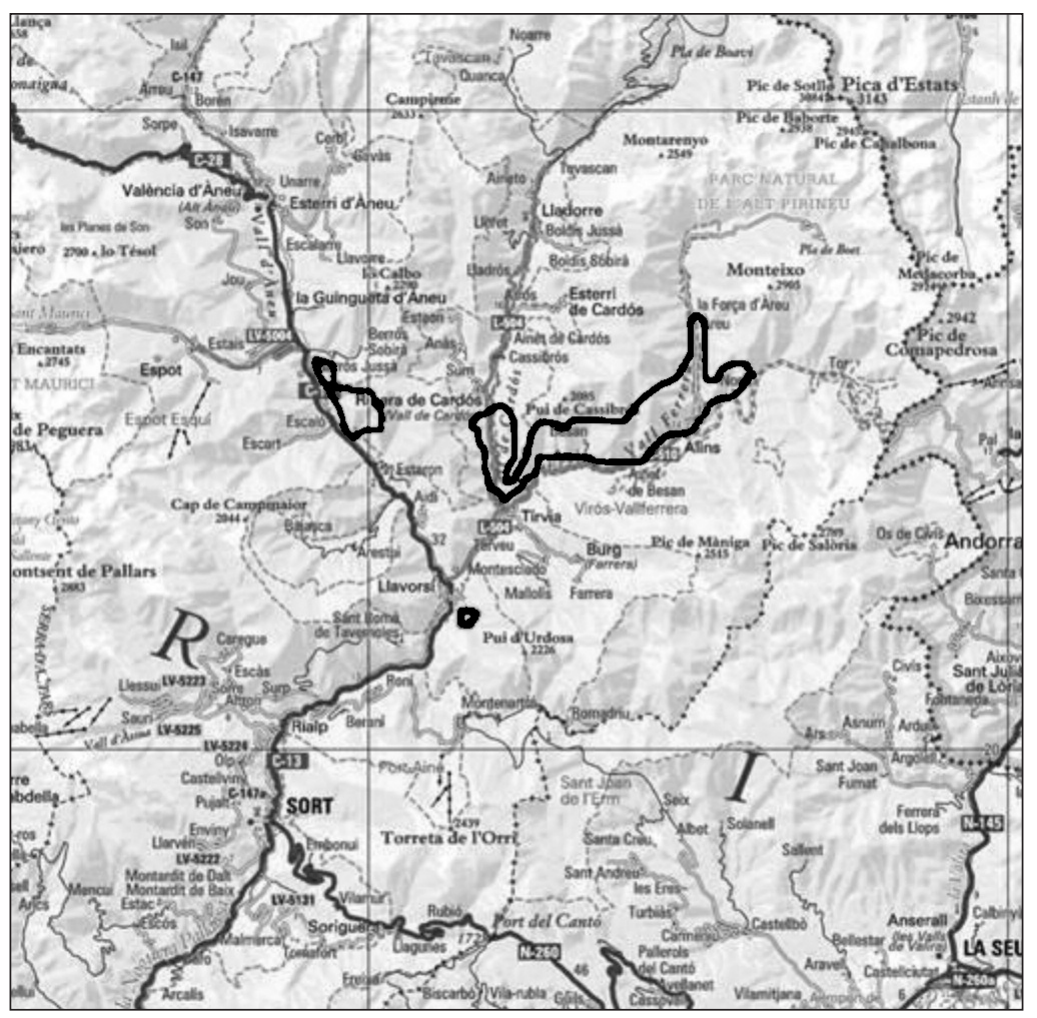

Figure 1 - Carte de répartition du Genévrier thurifère (Juniperus thurifera) en Haut Pallars (vallée de la Noguera Pallaresa, Catalogne, Espagne).

Figure 1 - Distribution map of the Spanish Juniper (Juniperus thurifera) in Haut Pallars (Noguera Pallaresa Valley, Catalonia, Spain).
Guerby (1993) a repéré une nouvelle population au Quié de Lujat (Ariège). L'absence notable de cet arbre méditerranéen en versant sud de la chaîne frontière avait toujours frappé les botanistes espagnols, qui cherchaient des localités intermédiaires entre celles du versant nord et les effectifs de la Dépression de l'Ebre (provinces de Zaragoza et Huesca) afin d'établir un lien biogéographique.

Au cours du XXI ${ }^{\mathrm{e}}$ siècle, seuls quelques arbres ou petits groupes d'arbres ont été recensés sur le piémont aragonais des pré-Pyrénées (Rodríguez-Ochoa \& Pedrol 1999 ; Ferrández 2003). Finalement, quelques noyaux représentatifs de Juniperus thurifera L. ont été découverts et délimités à partir de 2006 en Haut Pallars (Pyrénées centrales catalanes, Aymerich 2007). Centrés au Vall Ferrera, ils touchent aussi le Vall de Cardòs et deux points de la vallée principale du Noguera Pallaresa, de part et d'autre de Llavorsí (figure 1). Ce sont des bosquets épars dont la surface discontinue atteint 356 ha. Le nombre total de pieds est estimé entre 3600 et 4200 . Même si leur aire de répartition couvre sur la carte 37 carrés UTM de $1 \mathrm{~km}$ de côté, ils ne constituent sans doute qu'une seule population fonctionnelle (Aymerich 2008).

Nous présentons ici quelques données phytotopographiques, floristiques et écologiques concernant ces peuplements, de façon à signaler leur rôle dans le paysage végétal actuel. Cela va nous porter à une courte discussion sur leur possible origine biogéographique et à cerner leur état de conservation afin d'estimer leur évolution future.

\section{Matériel et méthodes}

Après avoir étudié la composition floristique des populations de Genévrier thurifère, nous avons dressé un tableau de vingt relevés où les espèces ont été ordonnées par groupes écologiques.

Quelques profils phytotopographiques représentatifs ont été réalisés, dans le but de mieux concrétiser l'exposition, la nature et profondeur du sol, les aspects topoclimatiques et les contacts géobotaniques avec les séries de végétation des alentours. La consultation de la bibliographie botanique, géobotanique et paléoécologique récente nous permet d'analyser les rapports de ces formations avec celles du versant nord de la chaîne et de la 
Dépression de l'Èbre, bassin versant auquel appartient la Noguera Pallaresa.

Enfin, nous avons évalué les affinités chorologiques et les types biologiques des plantes inféodées aux junipéraies, ainsi que les impacts de l'exploitation humaine, ce qui est important pour le Parc natural de l'Alt Pirineu et les services forestiers, organismes chargés de leur gestion conservatoire.

\section{Place des formations de \\ $J$. thurifera au sein du paysage végétal actuel du Pallars}

\section{Phytotopographie des communautés de J. thurifera}

Les différents profils (figures 2, 3 et 4) nous montrent les différentes situations de $J$. thurifera dans le Vall Ferrera, où sont concentrés la plupart des peuplements du Pallars. Dans la vallée inférieure (figure 2), les versants sont dominés par le Chêne vert, de sorte que notre arbre reste anecdotique entre 900-1300 m, précisément là où $J$. phoenicea $\mathrm{L}$. abonde sur les fentes ou les replats. Néanmoins, J. thurifera devient plus fréquent entre 1300-1 $400 \mathrm{~m}$ d'altitude. Dans la vallée moyenne (figure 3), le Chêne vert n'arrive que rarement à former des bosquets et $J$. phoenicea est à peu près inexistant. $J$. thurifera prend un rôle plus important sur les pentes rocheuses (adret d'Alins). Lorsqu'on pénètre vers les parties internes de la vallée, pas loin d'Àreu (figure 4), le Genévrier thurifère reste isolé et sa rareté est à relier à la diminution du nombre de versants exposés au sud et à l'augmentation de l'altitude moyenne. En amont d'Àreu, le Genévrier thurifère est absent.

$$
\begin{aligned}
& \text { Légende des figures 2, } 3 \text { et } 4 \text { : } \\
& \emptyset \text { Juniperus thurifera } \triangle \text { Juniperus phonica } \\
& \triangle \text { Pinus unclinata } \quad \text { Cytisus oromediterraneus } \\
& \triangle \text { Pinus sylvestris A. Vegetación rupicola } \\
& \text { Oraxinus excelsior } \quad m \text { Prados de siega } \\
& \text { Q Quercus pubescens rrr Pastos mesófilos } \\
& \text { Q Alnus glutinosa mi Pastos xerófilos } \\
& \text { Betula pendula } \ldots \text { Pastos supraforestales } \\
& \text { Quercusilex }
\end{aligned}
$$

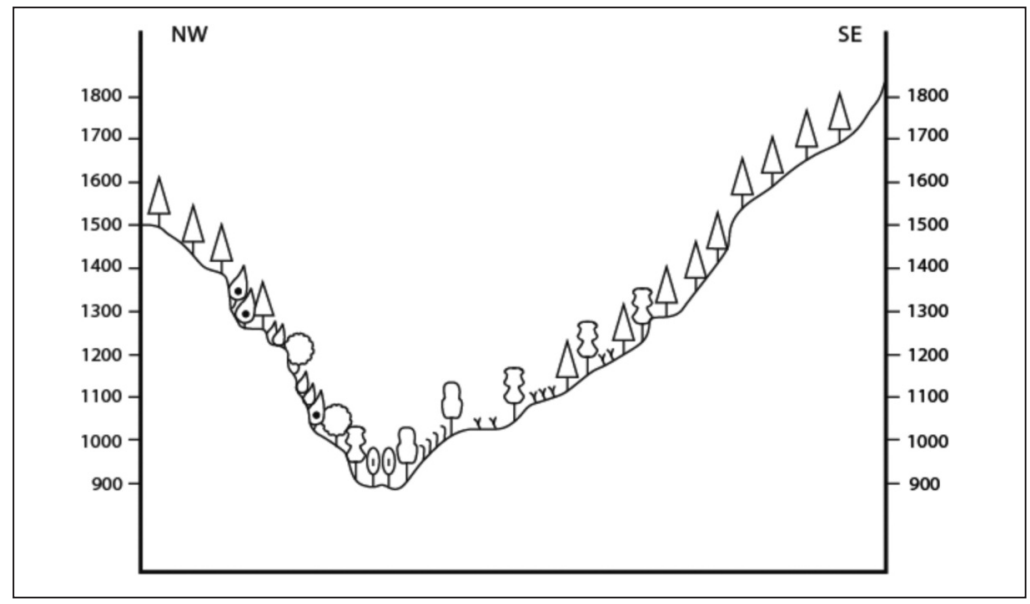

Figure 2 - Profil phytotopographique dans la vallée inférieure du Vall Ferrera, en montrant $J$. thurifera et $J$. phoenicea.

Figure 2 - Vegetation transect in the lower Vall Ferrera showing J. thurifera and J. phoenicea.

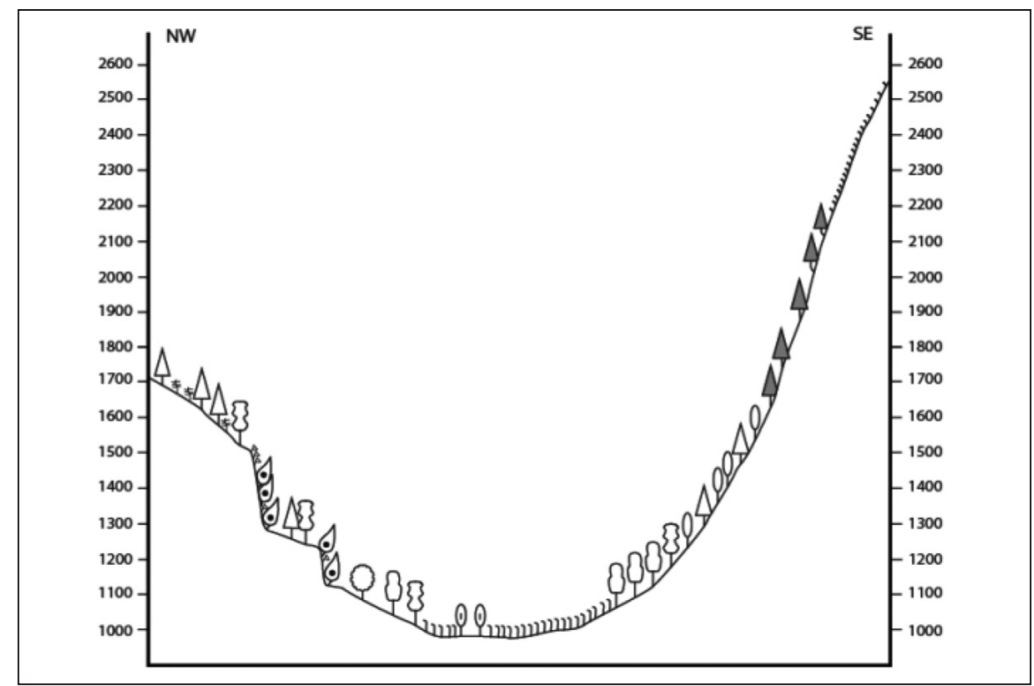

Figure 3 - Profil phytotopographique dans la vallée moyenne du Vall Ferrera, à la soulane d'Alíns, le plus important des peuplements de J. thurifera en Haut Pallars.

Figure 3 - Vegetation transect in the middle Vall Ferrera - southern slopes of Alins -, the best population of J. thurifera in the Haut Pallars.

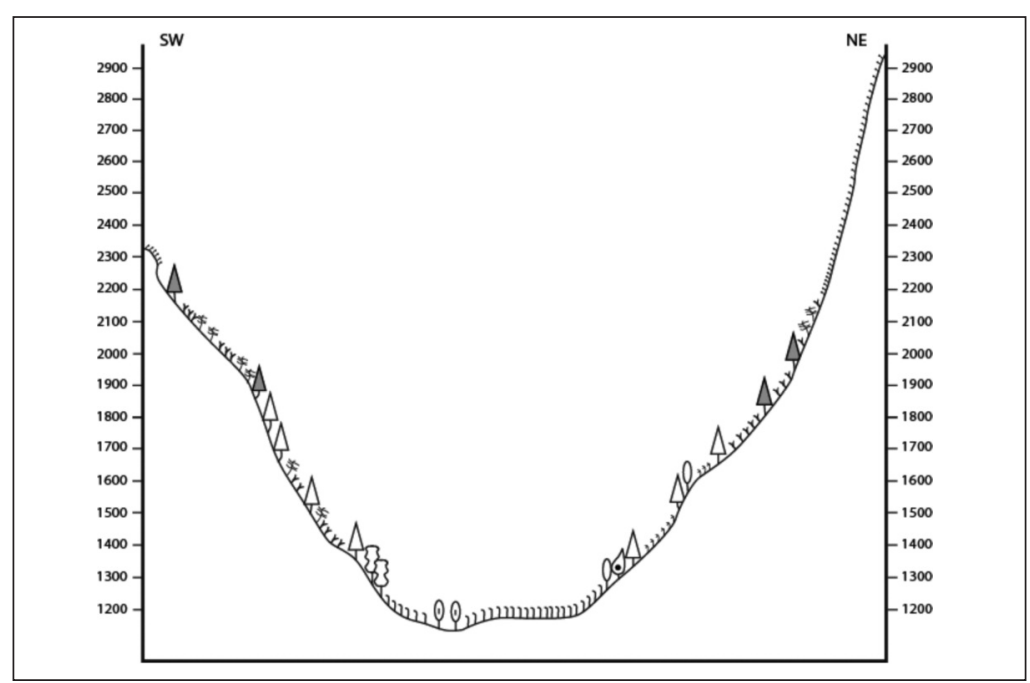

Figure 4 - Profil phytotopographique à l'intérieur du Vall Ferrera, près d'Àreu, avec des pieds isolés de $J$. thurifera.

Figure 4 - Vegetation transect in the interior of the Vall Ferrera, near Àreu, with isolated feet of J. thurifera. 


\section{Première interprétation des facteurs conditionnant la répartition des peuplements}

Les peuplements de Genévrier thurifère font partie de la végétation en mosaïque qui colonise les versants rocailleux très ensoleillés, de nature schisteuse, où l'on rencontre en mélange des espèces chasmophytes, des pelouses plus ou moins xérophiles et des plantes liées aux landes et groupements forestiers fragmentés. Des individus isolés se rencontrent également parmi les formations claires de Chêne vert (Quercus ilex L. subsp. ballota (Desf.) Samp.) sur ces mêmes replats et fentes. Enfin notre arbre peut également s'associer avec le Chêne pubescent [Q. humilis Miller (=Q. pubescens Willd.)], notamment sur les éboulis plus ou moins stabilisés.

La répartition actuelle de l'espèce ainsi que sa persistance dans la période historique seraient le résultat de la combinaison de facteurs climatiques et édaphiques ayant contrôlé l'expansion des essences ou espèces arbustives environnantes. Des conditions de conservation analogues ont été signalées à propos des peuplements marginaux du Genévrier dans les Hautes-Alpes et dans les monts Cantabriques (Garraud \& Villaret 2000 ; Bertrand \& Bertrand 2000).

À l'échelle régionale, il faut noter que le climat ne serait pas à lui seul un facteur déterminant pour notre arbre, étant donné que d'autres vallées des Pyrénées montrent une nuance plus continentale que le Pallars et que le thurifère ne s'y trouve pas. En effet, comme nous allons le voir, les espèces végétales nettement continentales nous manquent, tandis que les essences mésophiles telles que le hêtre (Fagus sylvatica L.) ou encore d'autres comme les fougères thermophiles Cheilanthes acrostica (Balbis) Tod. et $C$. tinaei Tod. peuvent être rencontrées aux alentours. Il est cependant vrai que le froid hivernal aurait néanmoins limité l'expansion en altitude d'espèces compétitives mais sensibles comme le Genévrier de Phénicie (Juniperus phoenicea).

Ce serait le topoclimat qui serait déterminant, car à l'échelle locale les pentes raides rocheuses provoquent en été une très forte évapotranspiration difficile à réduire par les arbres ou arbustes du domaine. Comme c'est le cas pour la plupart des peuplements situés en limite nord de son aire de répartition (monts Cantabriques, Pyrénées de HauteGaronne et Ariège, certaines stations des
Hautes-Alpes), il s'agit ici de bosquets plus ou moins rupicoles. La roche mère n'est pas calcaire mais métamorphique : calcschistes ou pélites paléozoïques très fissurés et instables dont l'inclination de $60-90 \%$ ne permet pas l'élaboration de sols profonds bien structurés. Ainsi, la succession vers les formations denses est difficile et le biotope n'est pas favorable aux espèces saxicoles car de petites variations du microrelief facilitent l'installation de plantes acidiphiles à côté des basiphiles. Dans ces conditions, les communautés permanentes deviennent favorables à la conservation d'espèces peu compétitives. La présence d'autres espèces du même genre ne serait due qu'au hasard. Ainsi J. phoenicea forme de belles populations sur le bas des versants plus abrités. J. sabina L., à l'opposé, reste plus localisé au secteur plus élevé d'Espot (Parc national d'Aigüestortes) et $J$. oxycedrus L. subsp. badia (H. Gay) Debeaux n'arrive à former que quelques stations en aval, entre Llavorsí et Rialp.

Toutefois, si le topoclimat a empêché l'installation des masses forestières de Pinus sylvestris L., Abies alba Miller, Quercus humilis, $Q$. subpyrenaica E.H. del Villar et $Q$. petraea Mattuschka, sûrement la concurrence avec $Q$. ilex subsp. ballota et avec Juniperus phoenicea aurait été plus forte, ces deux dernières espèces colonisant sans problème les versants rocheux des calcschistes. Finalement, la dominance potentielle du buis (Buxus sempervirens L., espèce calcicole) et du Genêt à balais (Cytisus oromediterraneus Rivas-Mart. et al., silicicole) sur certains endroits rocheux a été sans doute réduite en raison de la complexité des conditions édaphiques (variations du $\mathrm{pH}$ ) évoquées précédemment. Enfin, la diminution de l'écobuage répété aurait défavorisé ces deux arbustes plus ou moins pyrophiles.

\section{Composition floristique de la communauté}

Il ressort de cette étude une flore assez typique de l'étage montagnard des Pyrénées centrales continentales, entre le val d'Aran à l'ouest et l'Andorre à l'est, qui renferme quelques endémismes comme l'œillet des Pyrénées Dianthus pyrenaicus Pourr.

Le nombre d'espèces vasculaires qui accompagnent $J$. thurifera sur les versants du Pallars s'élève à 150 (tableau 1), mais il est à noter 
Tableau 1 - Les vingt relevés des populations de J. thurifera étudiées.

Table 1 - Twenty relevees of the studied J. thurifera populations.

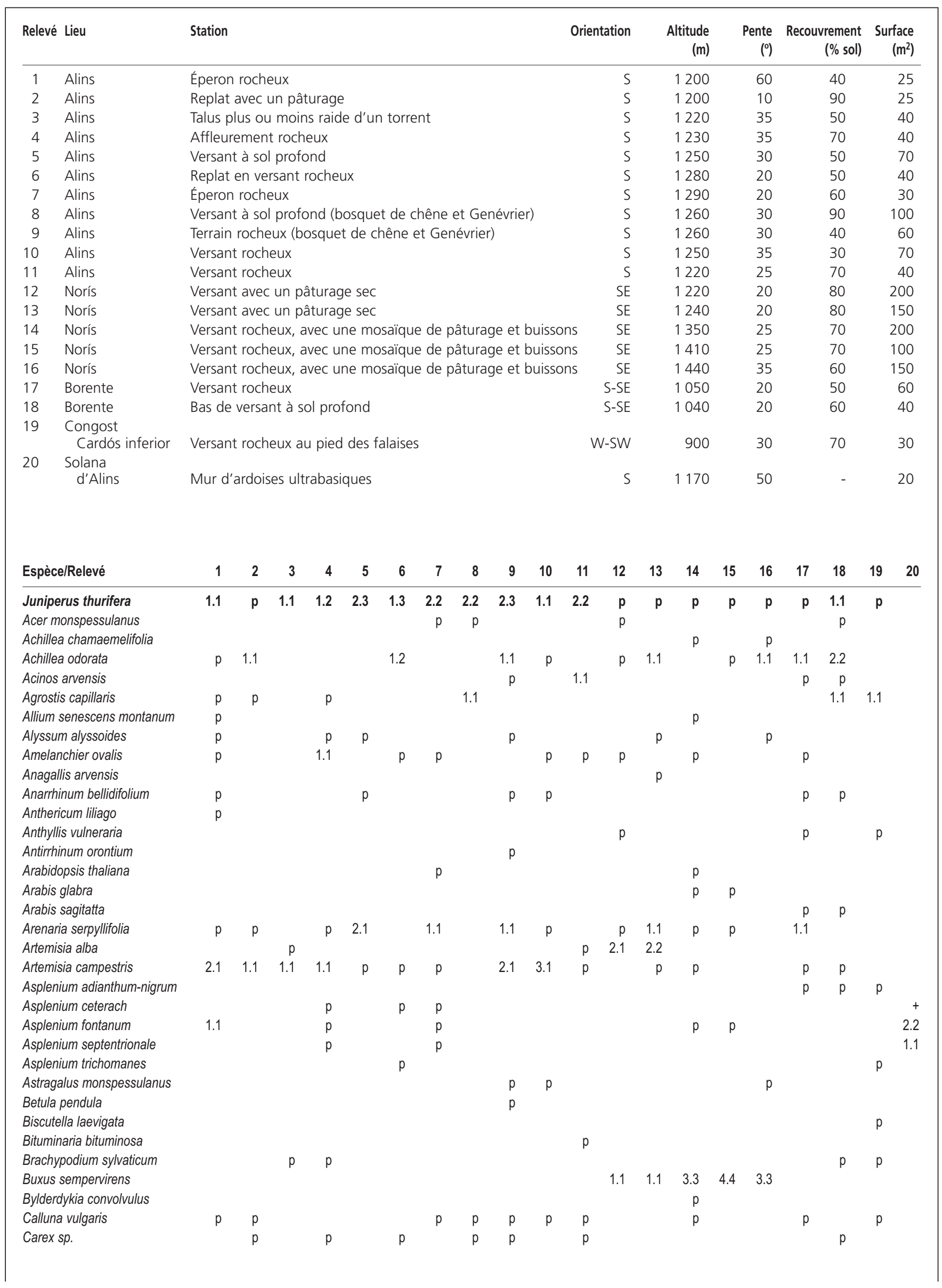




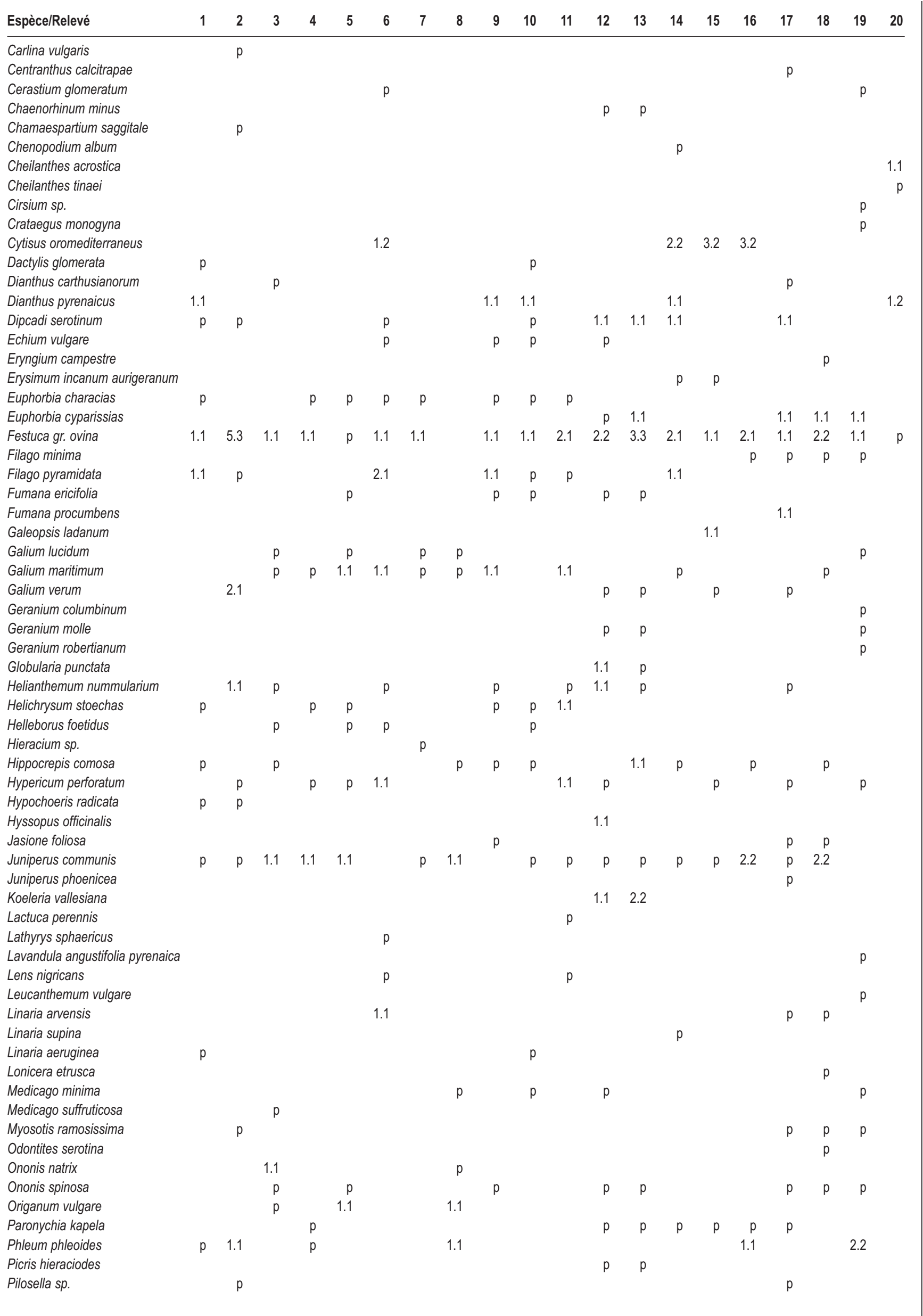




\begin{tabular}{|c|c|c|c|c|c|c|c|c|c|c|c|c|c|c|c|c|c|c|c|c|}
\hline Espèce/Relevé & 1 & 2 & 3 & 4 & 5 & 6 & 7 & 8 & 9 & 10 & 11 & 12 & 13 & 14 & 15 & 16 & 17 & 18 & 19 & 20 \\
\hline Pinus sylvestris & & & & & $p$ & & & & & & & & & & & & & & $p$ & \\
\hline Plantago lanceolata & & $\mathrm{p}$ & & & & & & & & & & & $\mathrm{p}$ & & & & & $p$ & & \\
\hline Plantago sempervirens & $\mathrm{p}$ & & $p$ & & & & & & $\mathrm{p}$ & & & & & & & & & $p$ & & \\
\hline Polygala nicaeensis & & $\mathrm{p}$ & & & & & & & & & & $p$ & & & & & & $p$ & & \\
\hline Potentilla neumanniana & & & & & & & & & & & & $\mathrm{p}$ & & & & & $\mathrm{p}$ & $p$ & & \\
\hline Prunus mahaleb & & & & & & & & & & & & & & & & & & $p$ & & \\
\hline Prunus spinosa & & 2.2 & & & 1.1 & & $p$ & & & & & & $\mathrm{p}$ & & & & & 1.1 & $\mathrm{p}$ & \\
\hline Ptychotis saxifraga & & & & & & & & & & & & & & & & & & & $\mathrm{p}$ & \\
\hline Quercus ilex & $\mathrm{p}$ & $p$ & & & & & & & & & & & & & & & $\mathrm{p}$ & $p$ & $p$ & \\
\hline Quercus pubescens & & $p$ & $p$ & 2.3 & $p$ & $\mathrm{p}$ & 2.2 & 4.5 & 2.2 & 2.2 & 2.2 & & & & & & $\mathrm{p}$ & $p$ & & \\
\hline Ranunculus bulbosus & & & & & & & & & & & & & $\mathrm{p}$ & & & & & & $\mathrm{p}$ & \\
\hline Reseda phytheuma & & & & & & & & & & & & $p$ & & & & & & & & \\
\hline Rhamnus alaternus & $p$ & & & 1.1 & & & & & & & & & & & & & & & & \\
\hline Rumex acetosella & & & & & & & & & & & & & & & & $p$ & & & & \\
\hline Rorippa pyrenaica & & & & & & & & & & & & & & $p$ & & & & & & \\
\hline Rosa agrestis & & & & & & & & & & & $\mathrm{p}$ & & & & $p$ & & & & & \\
\hline Rosa canina & & & & $p$ & & & & & & & & & $p$ & & & & & & & \\
\hline Rosa sp. & & & & & $\mathrm{p}$ & $\mathrm{p}$ & $\mathrm{p}$ & $p$ & & & & $\mathrm{p}$ & & & & & $\mathrm{p}$ & & & \\
\hline Rubus ulmifolius & & & 1.1 & $\mathrm{p}$ & 1.1 & & & $\mathrm{p}$ & & & & & & & & & & & 1.1 & \\
\hline Sanguisorba minor & $p$ & & $p$ & & & & $p$ & & & & & $p$ & $\mathrm{p}$ & $p$ & & & $\mathrm{p}$ & & $p$ & \\
\hline Santolina chamaecyparissus & $\mathrm{p}$ & & & & & & & & & & & & & & & & $\mathrm{p}$ & & & \\
\hline Saponaria ocymoides & & & $p$ & $p$ & 1.1 & $\mathrm{p}$ & & & 2.1 & $\mathrm{p}$ & & & & & & & & & & \\
\hline Satureja montana & $\mathrm{p}$ & $p$ & $p$ & $\mathrm{p}$ & $p$ & 1.1 & $p$ & & & $\mathrm{p}$ & $p$ & 1.1 & 2.2 & & & & & & $\mathrm{p}$ & \\
\hline Scabiosa columbaria & & & & & & & $p$ & & & & & & & & & & $\mathrm{p}$ & & $p$ & \\
\hline Scleranthus annuus & & 2.1 & & & & & & & & & & & & & & & & & & \\
\hline Scleranthus polycarpos & & & & & & & & & & $\mathrm{p}$ & & & & 1.1 & $p$ & $\mathrm{p}$ & $\mathrm{p}$ & $p$ & & \\
\hline Sedum album & $\mathrm{p}$ & & $p$ & $p$ & & & & & $\mathrm{p}$ & $\mathrm{p}$ & & $\mathrm{p}$ & $\mathrm{p}$ & $p$ & & & 1.1 & $p$ & 1.1 & \\
\hline Sedum brevifolium & & & & & & & & & & & & & & & & & $p$ & & & $p$ \\
\hline Sedum dasyphyllum & 1.1 & & & $p$ & & & $p$ & & $p$ & $p$ & & $p$ & & $p$ & & & & & & 1.1 \\
\hline Sedum montanum & & & & & & & & & & & & & & & & & $\mathrm{p}$ & $p$ & $\mathrm{p}$ & \\
\hline Sempervivum arachnoideum & 2.1 & & & $\mathrm{p}$ & & & & & & $\mathrm{p}$ & & $\mathrm{p}$ & & $\mathrm{p}$ & & & & & & 1.2 \\
\hline Sempervivum tectorum & & & & & & & & & & & & & & $\mathrm{p}$ & & & & & $\mathrm{p}$ & \\
\hline Seseli montanum & $p$ & $p$ & & & & $\mathrm{p}$ & & & & & $\mathrm{p}$ & $\mathrm{p}$ & & $p$ & $\mathrm{p}$ & & $\mathrm{p}$ & & & \\
\hline Silene nutans & $\mathrm{p}$ & & & & & & & & & $\mathrm{p}$ & & & & & & $\mathrm{p}$ & $\mathrm{p}$ & & & \\
\hline Silene saxifraga & & & & & & & $p$ & & & & & & & $p$ & & & & & & $p$ \\
\hline Stachys recta & $\mathrm{p}$ & $\mathrm{p}$ & & & & & & & $\mathrm{p}$ & $\mathrm{p}$ & $\mathrm{p}$ & $\mathrm{p}$ & & & & & $\mathrm{p}$ & 1.1 & & \\
\hline Stipa pennata eriocaulis & & & & & & & & & & & & 4.3 & $\mathrm{p}$ & & & & & 2.1 & & \\
\hline Teucrium chamaedrys & & & & & $\mathrm{p}$ & $\mathrm{p}$ & & 1.1 & 1.1 & $p$ & & 1.1 & $p$ & & $\mathrm{p}$ & & 1.1 & 1.1 & 1.1 & \\
\hline Teucrium scorodonia & & & & & & & & & & & & & & & & & & & $p$ & \\
\hline Thesium divaricarum & & $p$ & & & & & & & & & & & & & & & & & & \\
\hline Thymus polytrichus & 2.1 & $p$ & 1.1 & $\mathrm{p}$ & & 1.1 & $p$ & & 1.1 & 2.1 & 1.1 & $\mathrm{p}$ & $\mathrm{p}$ & 1.1 & & & $\mathrm{p}$ & $p$ & $p$ & $p$ \\
\hline Thymus vulgaris palearensis & & & & & & & & & & & & & & & & & 2.2 & 2.1 & & \\
\hline Trifolium arvense & & 1.1 & & $\mathrm{p}$ & & $\mathrm{p}$ & & $\mathrm{p}$ & $p$ & & & & & & & & 1.1 & 2.1 & $\mathrm{p}$ & \\
\hline Trifolium campestre & & $p$ & & & & $\mathrm{p}$ & & & & & & & & & & & $\mathrm{p}$ & $p$ & & \\
\hline Trifolium scabrum & & & & & & & & & & & & & & & & & $p$ & $p$ & & \\
\hline Umbilicus rupestris & $\mathrm{p}$ & & & & & & $p$ & & & & & & & $p$ & $p$ & & & & & \\
\hline Valerianella sp. & & & & & & & & & & & & & $\mathrm{p}$ & & & & & & & \\
\hline Verbascum sp. & & $p$ & & & & & & & & & & & & $p$ & & & & & & \\
\hline Veronica austriaca & & $\mathrm{p}$ & & & & $\mathrm{p}$ & & & $\mathrm{p}$ & & & $\mathrm{p}$ & $\mathrm{p}$ & & & & $\mathrm{p}$ & $p$ & & \\
\hline Veronica verna & & $p$ & & & & & & & & & & & & & & & $\mathrm{p}$ & $p$ & $p$ & \\
\hline Vicia angustifolia & & & & & & & & $\mathrm{p}$ & & $\mathrm{p}$ & $\mathrm{p}$ & & & & & & $\mathrm{p}$ & $p$ & & \\
\hline Vicia lutea & & & & & & & & & & & & $\mathrm{p}$ & & & & & & & & \\
\hline Vicia parviflora & & $p$ & $p$ & $p$ & $\mathrm{p}$ & & & & & $\mathrm{p}$ & & & & & & & & & $\mathrm{p}$ & \\
\hline Vincetoxicum nigrum & & & & $p$ & & & & & & $p$ & & & & & & & & & & \\
\hline
\end{tabular}

que les $93,3 \%$ n'apparaissent que dans $50 \%$ ou moins des relevés. La plupart de ces espèces n'est donc que légèrement inféodée à notre arbre et par conséquent la composition floristique varie beaucoup d'une localité à l'autre. Les espèces les plus fidèles sont Thymus polytrichus A. Kerner et Festuca gr. ovina L. dont la présence est observée dans plus de $75 \%$ des relevés. Parmi les espèces fréquentes, seulement trois se montrent abon- 
Tableau 2 - Espèces les plus fréquentes et les plus abondantes contenues dans nos relevés de $J$. thurifera.

Table 2 - The more frequent and the more abundant species in the studied relevees of $\mathrm{J}$. thurifera.

\begin{tabular}{ll}
$\begin{array}{l}\text { Espèces les plus fréquentes } \\
\text { (présentes dans plus } \\
\text { de } \mathbf{5 0} \% \text { des relevés) }\end{array}$ & $\begin{array}{l}\text { Espèces localement abondantes } \\
\text { (recouvrement }>\mathbf{5 0} \% \text { au moins } \\
\text { dans un relevé) }\end{array}$ \\
\hline Achillea odorata L. & $\begin{array}{l}\text { Artemisia campestris L. } \\
\text { Auxus sempervirens L. } \\
\text { Arenaria serpyllifolia L. }\end{array}$ \\
$\begin{array}{l}\text { Artemisia campestris L. } \\
\text { Festuca gr. ovina L. }\end{array}$ & Festuca gr. ovina L. \\
Juniperus communis L. & Quercus humilis Miller \\
Quercus humilis Miller & Stipa pennata L. \\
Satureja montana L. & \\
Sedum album L. & \\
Teucrium chamaedrys L. & \\
Thymus polytrichus A. Kerner &
\end{tabular}

dantes dans quelques points (tableau 2): Artemisia campestris L., Festuca gr. ovina et Quercus humilis, mais généralement elles ne sont pas dominantes et leur importance est équivalente à celle de $J$. thurifera là où elles cohabitent.

Ces conditions déterminent des groupements hétérogènes où une bonne partie des espèces profite des vides laissés par les plantes dominantes aux endroits les moins favorables à celles-ci. Il est à souligner que la même idée de «faible homogénéité » avait été déjà émise

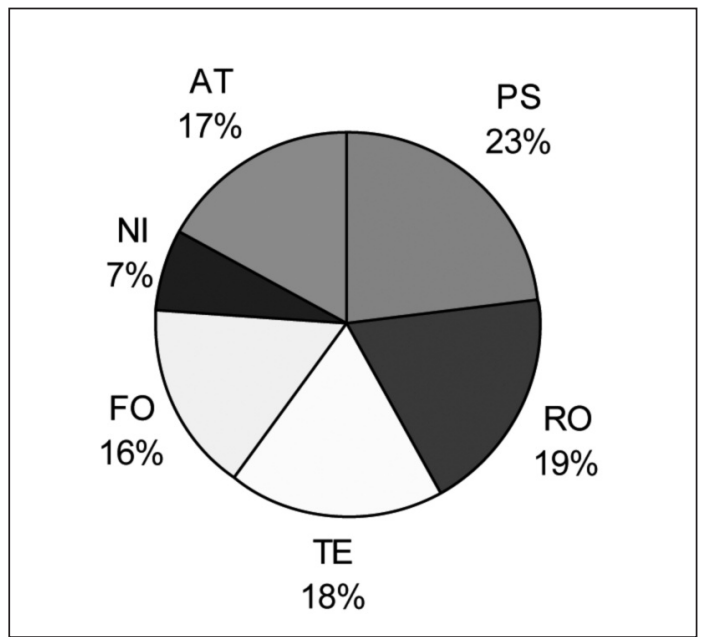

Diagramme 1 - Écologie des espèces compagne (\%). PS : pelouses xérophiles médioeuropéennes; RO : habitats rocheux; TE : thérophytes non nitrophiles; FO : habitats forestiers ; NI : nitrophiles; AT : autres.

Diagram 1 - Ecological preferences of the accompanying species (\%). PS: dry pastures of Middle Europe ; RO: rocky habitats; TE: therophytes non nitrophiles ; FO: forestal habitats ; NI: nitrophiles ; AT: others. par Braun-Blanquet \& Bolòs (1957) après leur étude des peuplements de l'Èbre moyen, très différents des forêts de Genévrier thurifère du centre de son aire ibérique où s'observent plus de dix associations bien définies (Rivas-Martínez et al. 2011).

Ainsi, les espèces du cortège floristique du Genévrier thurifère ont des affinités écologiques et biogéographiques très variées (diagramme 1). Çà et là les plantes liées aux pelouses dites xérophiles eurosibériennes peuvent être abondantes, de même que les rupicoles, les plantes de pâturages à thérophytes ou encore les plantes forestières. Au sein de chacun des deux derniers groupes, on peut rencontrer des espèces eurosibériennes, méditerranéennes ou plurirégionales, tandis que chez les plantes némorales, la composante eurosibérienne atteint $60 \%$. Enfin la présence significative d'espèces nitrophiles $(7 \%)$ doit être reliée à l'élevage ancien et récent que l'on a pratiqué sur ces territoires voués à la transhumance (Violant 2001).

En ce qui concerne les types biologiques, la plupart des espèces notées sont herbacées (65\%), avec une dominance claire des hémicryptophytes et une proportion non négligeable de thérophytes (diagramme 2). Parmi les espèces ligneuses les chaméphytes se montrent plus fréquentes que les phanéro-

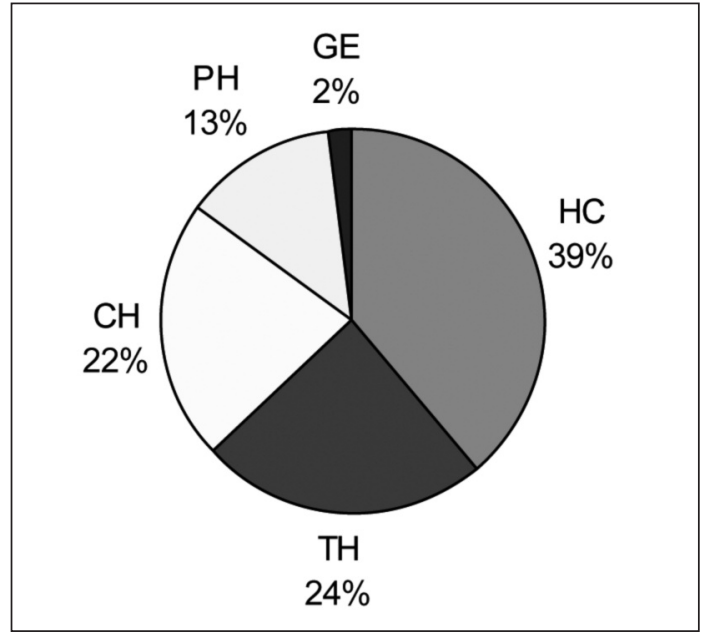

Diagramme 2 - Types biologiques des espèces compagnes (\%). HC : hémicryptophytes; TH : thérophytes; $\mathrm{CH}$ : chaméphytes : PH : phanérophytes; GE : géophytes.

Diagram 2 - Biological types of accompanying species (\%). HC: hemicryptophytes; TH: therophytes; CH: chamaephytes ; PH: phanerophytes; GE: geophytes. 
phytes. Cette répartition se retrouve dans les thuriféraies des Alpes où les hémicryptophytes dominent (Garraud \& Villaret 2000), même si le chiffre des thérophytes est plus bas $(6,4 \%)$, sans doute en raison d'une influence climatique méditerranéenne moins forte que dans les Pyrénées du Pallars. Les îlots de thuriféraie, placés entre les domaines eurosibériens et méditerranéens, ne seraient donc qu'une forme singulière extrême de cette dernière, à classer très probablement dans l'Alliance Juniperion thuriferae Rivas-Martínez 1969 de la classe Junipero sabinae-Pinetea ibericae qui inclut les peuplements de la Dépression moyenne de l'Èbre.

\section{Discussion}

\section{Origine et évolution des peuplements}

L'aire de répartition fragmentée de Juniperus thurifera avec quelques-unes de ses taches en régression pose beaucoup de problèmes (Gauquelin et al. 1999) quand on veut établir des rapports et affinités entre les différentes populations, d'autant plus que les successives approximations obtenues en suivant des méthodologies différentes ne se montrent pas coïncidentes (Gauquelin et al. 1988 ; Barbero et al. 1994 ; Adams et al. 2003 ; Jiménez et al. 2003 ; Marcysiak et al. 2007 ; Terrab et al. 2008 ; Rivas-Martínez et al. 2011). Pour Terrab et al. (2008), l'origine des peuplements corses aurait été facilitée par la dispersion sporadique des graines à longue distance, mais les populations isolées ou petites auraient un caractère relique, surtout en limite nord de leur aire actuelle: monts Cantabriques, Pyrénées, Alpes et Corse (Losa \& Montserrat 1953 ; Romero 1983 ; Barbero et al. 1987 ; Barbero \& Quézel 1996 ; Gamisans 2000 ; Garraud \& Villaret 2000 ; Bertrand \& Bertrand 2000 ; Gauquelin et al. 2003 ; Aymerich 2007).

En se basant sur les données polliniques et concernant la présence de Juniperus sp., Jalut et al. (2000a, 2000b) proposaient, pour les peuplements pyrénéens, l'hypothèse d'une colonisation postglaciaire (14 000-13 000 BP) expansive de $J$. thurifera, à la faveur d'un climat de type méditerranéen continental caractérisé par des hivers froids et des étés plus ou moins arides. Cela n'impliquerait pas nécessairement une colonisation à longue distance, car la présence de pollen de Juniperus sp. est attestée au Pourtalet (Pyrénées aragonaises) de façon continue mais avec des fluctuations entre 30000 et $14000 \mathrm{BP}$, toujours en compagnie d'espèces à affinité steppique (González-Sampériz et al. 2006). Pendant une grande partie de la dernière période glaciaire, les territoires périphériques des Pyrénées espagnoles auraient été couverts par des formations herbacées steppiques parsemées de genévriers, comme on peut les observer aujourd'hui dans les domaines oro-méditerranéens du Système Ibérique, dominés par J. thurifera et J. sabina. Avant 20000 BP, dans les pré-Pyrénées, près de la Dépression de l'Èbre, les mêmes auteurs décrivent une steppe à conifères avec Pinus sp. et Juniperus sp., puis entre 20000 et $10000 \mathrm{BP}$ une augmentation des conifères et une légère expansion en altitude des caducifoliés. Les forêts actuelles se mettent en place à partir de 10000 ans.

Concernant les Juniperus, on peut supposer qu'au cours de ces périodes, dans les zones les plus froides, dominaient J. communis, $J$. thurifera et $J$. sabina, tandis qu'à plus basse altitude J. oxycedrus et J. phoenicea s'ajoutaient aux $J$. thurifera. Cette mosaïque s'est maintenue au cours des 10000 dernières années uniquement dans les dépressions semiarides de la vallée moyenne de l'Èbre. Cette forte extension passée avait été envisagée par Braun-Blanquet \& Bolòs (1957) et Montserrat (1966) lors de leurs études sur les stations aragonaises actuelles et cette hypothèse est renforcée par la découverte récente des petites stations périphériques (Aparicio et al. 1993 ; Ferrández 2005 ; Arizaleta et al. 2008).

Pendant la dernière période glaciaire et le Tardiglaciaire, plusieurs espèces de Juniperus auraient donc pu jouer un rôle plus important qu'aujourd'hui (Valero-Garcés et al. 2004 ; González-Sampériz et al. 2005). Ultérieurement, surtout dans les Monegros, une augmentation de Pinus et une diminution parallèle de Juniperus sp. s'est produite vers 8600 BP (Davis 1994). Il est donc probable que nos populations du Genévrier thurifère du Pallars résultent d'une expansion postglaciaire à courte ou moyenne distance. Mais pendant la dernière glaciation, leur persistance in situ ne peut être exclue sur des zones topographiquement et climatiquement favorables.

Entre Monegros et Pallars, quelques peuplements intermédiaires auraient disparu pendant l'Holocène, du fait de la concurrence des 


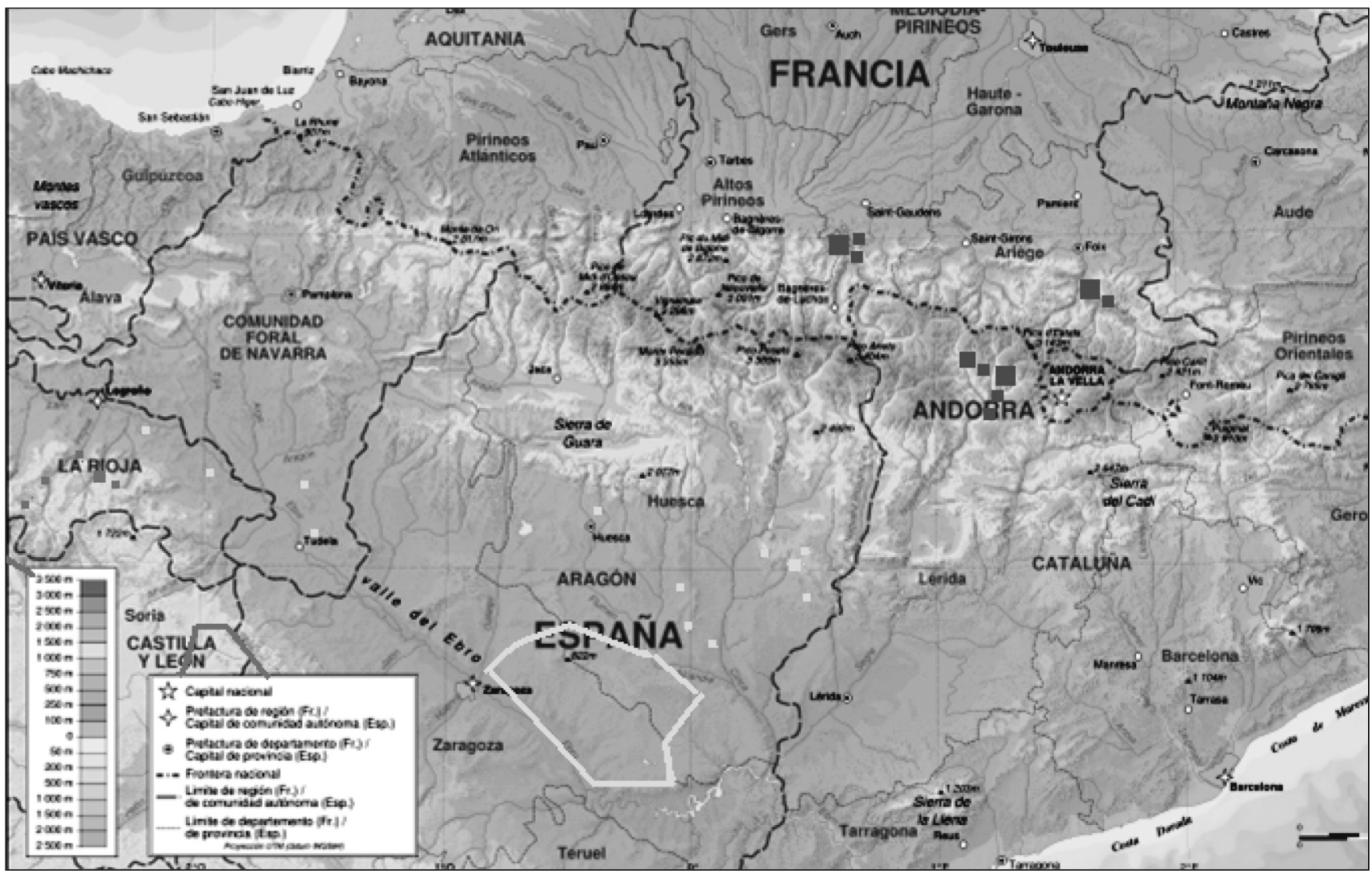

Figure 5 - Carte de répartition du Genévrier thurifère (Juniperus thurifera) dans la vallée de l’Èbre et les Pyrénées. Périmètre : aire de présence fréquente. Gros carrés : stations ponctuelles. Petits carrés : individus ou petits groupes. Code de couleurs: noir, Pyrénées ; gris clair, Dépression de l'Èbre ; gris foncé, Système Ibérique.

Figure 5 - Distribution map of the Spanish Juniper (Juniperus thurifera) in the Ebro Valley and the Pyrenees. Perimeter: area where the tree is frequent. Large squares: isolated stations. Small squares: individual or reduced groups. Colour code: black, Pyrenees ; light gray, Ebro Depression ; dark gray, Iberian System.

espèces mésoméditerranéennes en expansion depuis 10000 ans (González-Sampériz et al. 2005). Les petits groupes périphériques touchant les pré-Pyrénées (Rodríguez-Ochoa \& Pedrol 1999 ; Ferrández 2003) témoigneraient d'une aire de répartition moins discontinue dont les bosquets du Pallars seraient le dernier échelon.

Les données génétiques permettant de confirmer cette filiation manquent encore mais la présence fréquente au Pallars du diptère cecidiogène endémique Etsuhoa thuriferae, également abondant aux Monegros (BlascoZumeta 2000), est un argument positif. Cette petite mouche exclusive du Genévrier thurifère est également signalée dans le Système Ibérique et au Maroc (Skuhravá 1995). Sa présence au Pallars conforte l'hypothèse déjà rappelée d'une ancienne connexion entre les junipéraies sud-pyrénéennes, de la Dépression de l'Èbre et du Système Ibérique.

Toutefois, l'origine des trois populations pyrénéennes (deux en France, une en Espagne) pourrait être différente, malgré la courte dis- tance géographique qui les sépare. En effet, pour Terrab et al. (2008), la population de Marignac (Haute-Garonne) est à rapprocher génétiquement de celles des Alpes et d'Espagne centrale, mais elle s'éloigne de celles des Monegros. Les populations garonnaises pourraient donc résulter de la fragmentation d'une aire couvrant le piémont français des Pyrénées ou bien la dernière avant-garde d'une expansion est-ouest.

\section{Dynamique postglaciaire des populations du Pallars}

Au Pallars, le rôle du Genévrier thurifère dans le paysage végétal semble avoir été secondaire au cours des derniers 10000 ans. Les données paléobotaniques de la Coma de Burg située $6 \mathrm{~km}$ au sud des stations principales de thurifère et à une altitude plus élevée (1 800 m) (Pèlachs et al. 2001 ; Pèlachs 2004) montrent que vers 9400 BP les zones élevées étaient couvertes par une mosaïque de pâturages parsemés de noisetiers et bouleaux. Au 
fond des vallées dominaient les chênaies caducifoliées. Les chênes verts étaient déjà présents et de possibles concurrents pour le thurifère. Entre 10000 et $5000 \mathrm{BP}$, peut-être à cause de la densification des forêts subalpines, les Juniperus sp. étaient rares et disparaissent vers $8300 \mathrm{BP}$. C'est seulement vers $4500 \mathrm{BP}$, à la suite d'une augmentation de la pression anthropique sur les forêts qu'ils réapparaissent avec Artemisia sp. atteignant leur représentation maximale holocène.

Dans le Pallars, malgré une forte déforestation au Moyen Âge affectant les chênaies, les hêtraies et les sapinières, puis plus tard, pendant la révolution industrielle $\left(\mathrm{XVII}^{\mathrm{e}}\right.$ et $\mathrm{XVIII}^{\mathrm{e}}$ siècles), les nombreuses «forges à la catalane », les données palynologiques citées ne signalent pas une régression forte de $J$. thurifera. La toponymie nous confirme sa présence traditionnelle dans le Vall Ferrera. Le «Turó de la Savina Rodona » est au-dessus d'Ainet de Besan et la «Savinyeda » à Norís. Mais rien ne fait penser à une espèce déterminante dans le paysage végétal.

Les habitants âgés d'Alins connaissaient bien l'existence des genévriers, mais aujourd'hui personne ne se souvient de grands exemplaires ou de formations denses ayant pu donner lieu à des exploitations particulières, à l'inverse de ce qui existe à l'intérieur de la péninsule Ibérique où Juniperus thurifera constitue une ressource très appréciée (Villar \& Ferrández 2000). Ici, la disponibilité de nombreux chênes, pins, sapins, etc. aux alentours des villages a pu satisfaire les besoins locaux en bois.

Les notes et publications botaniques réalisées pendant le $\mathrm{XX}^{\mathrm{e}}$ siècle confirment une certaine stabilité de ces formations de genévrier. Font Quer (1915), illustre botaniste, visita le Vall Ferrera en 1911 en passant à quelques centaines de mètres des stations principales sans les repérer. Cela pourrait indiquer que les peuplements n'étaient alors ni plus denses ni plus abondants qu'aujourd'hui. On peut dire la même chose pour la seconde moitié du $\mathrm{XX}^{\mathrm{e}}$ siècle, malgré des travaux spécifiques concernant la carte forestière (Jordán de Urriés 1954), la flore (Farreny 1978) et la carte de végétation (Carreras et al. 2000). La plupart de nos genévriers sont confinés sur les rochers d'accès parfois difficile et quelquesuns ont été confondus avec $J$. phoenicea, tant leur présence était surprenante! Rappelons que pour Labatut et al. (2000), une semblable erreur s'est produite en Ariège...
Finalement, au cours des dernières décades une expansion limitée de J. thurifera s'est produite à la suite de l'abandon de l'agriculture et de l'élevage des chèvres (Aymerich 2008). Ce phénomène est observé d'une façon assez générale en Espagne et en France (Allende et al. 1999; Gauquelin et al. 1999; Gamisans 2000 ; Labatut et al. 2000).

On peut ainsi, aujourd'hui, observer de jeunes genévriers colonisant les champs ou les prairies abandonnées il y a 30 à 60 ans, de préférence au pied des versants rocheux où l'espèce est relativement fréquente. Ici comme dans les Alpes ou de l'autre côté des Pyrénées, nous avons affaire à des noyaux dits primaires sur sols pierreux et secondaires sur sols profonds (Borel \& Polidori 1983 ; Cambecèdes et al. 2005), ces derniers pouvant disparaître à long terme du fait de la concurrence avec d'autres essences.

\section{Conclusion}

Après avoir étudié la flore, la phytotopographie et la dynamique des peuplements isolés du Genévrier thurifère au Pallars, la stabilité de ses effectifs nous semble assurée, sous réserve de petits changements locaux. À notre avis, il n'y a aucun risque pour les noyaux principaux des terrains rocheux, sauf un incendie catastrophique. Seules quelques régressions ponctuelles peuvent concerner les peuplements les plus récents, précisément là où le développement d'autres essences concurrentielles devient possible, lorsque la pratique du feu pastoral a été interrompue. Heureusement, la valeur patrimoniale de ces bosquets de Juniperus thurifera est reconnue et leur gestion conservatoire assurée, car la plupart des effectifs appartiennent au Parc Natural de l'Alt Pirineu, déclaré par le gouvernement régional de Catalogne en 2003, et à l'échelle européenne ils ont été inclus dans le réseau Natura 2000 (directive Habitat).

\section{Remerciements}

Notre ami J.A. Sesé a figuré à l'ordinateur nos schémas phytotopographiques. T. Gauquelin et deux lecteurs anonymes ont corrigé le texte. Qu'ils en soient remerciés. 


\section{Références}

Adams R.P., Mumba L.E., Shelley S.A., Pandery R.M., Gauquelin T. \& Badri W., 2003. Geographic variation in the leaf oils and DNA fingerprints (RAPDs) of Juniperus thurifera L. from Morocco and Europe. Journal of Essential Oils Research 15: 148-154.

Allende F., Guerra J.C. \& López N., 1999. Dinámica reciente de las formaciones de Juniperus en el centro de la Península Ibérica. In: Actas del XVI Congreso de Geógrafos Españoles. Departamento de Geografía de la Universidad de Málaga.

Aparicio J.M., Patino S., Pérez Dacosta T., Uribe-Echebarría P.M., Urrutia P. \& Valencia J., 1993. Notas corológicas sobre la flora vascular del País Vasco y aledaños (VII). Estudios del Museo de Ciencias Naturales de Álava 8 : 85-99.

Arizaleta J.A., Fernández Aldana R., Quintana J.D., Alejandre J.A. \& Benito Ayuso J., 2008. La sabina albar (Juniperus thurifera L.) en la Rioja. Zubía 25-26: 7-16.

Aymerich P., 2007. Juniperus thurifera L. als Pirineus catalans. Butlletí de la Institució Catalana d'Història Natural 73 : 121-123.

Aymerich P., 2008. La savina turífera (Juniperus thurifera L.) als Pirineus Catalans : distribució, població i conservació. Orsis 23 : 9-26.

Barbero M. \& Quézel P., 1986. Place et rôle de Juniperus thurifera dans les structures de végétation des Alpes du Sud. Lazaroa 9 : 255-275.

Barbero M., Hammoud A. \& Quézel P., 1987. Le Genévrier thurifère dans les Alpes ligures. Bulletin Societé Linnéenne de Provence 39 : 75-79.

Barbero M., Lebreton P. \& Quézel P., 1994. Sur les affinités biosystématiques et phytoécologiques de Juniperus thurifera L. et du Juniperus excelsa Bieb. Ecologia Mediterranea 20 : 21-37.

Bertrand C. \& Bertrand G., 2000. Les juniperaies à Genévrier thurifère dans les géosystèmes cantabriques : analyse d'une « durabilité » (nord-ouest de la Péninsule Ibérique - provinces de León et de Palencia - Espagne). In : Gauquelin T., Asmodé J.F. \& Largier G. (eds.), Le Genévrier thurifère (Juniperus thurifera L.) dans le bassin occidental de la Méditerranée : systématique, écologie, dynamique et gestion. Actes du colloque international de Marignac (Haute-Garonne), 26-27 septembre 1997. Paris, ONF, coll. « Les dossiers forestiers » $6:$ 47-52.

Blasco-Zumeta J., 2000. Contribution à l'étude de la faune associée à Juniperus thurifera L. dans les Monegros (Aragón, Espagne). In : Gauquelin T., Asmodé J.F. \& Largier G. (eds.), Le Genévrier thurifère (Juniperus thurifera L.) dans le bassin occidental de la Méditerranée : systématique, écologie, dynamique et gestion. Actes du colloque international de Marignac (Haute-Garonne), 26-27 septembre 1997. Paris, ONF, coll. «Les dossiers forestiers » $6: 94-103$.

Borel A. \& Polidori J.L., 1983. Le Genévrier thurifère (Juniperus thurifera L.) dans le Parc national du Mercantour (Alpes-Maritimes). Bull. Soc. Bot. Fr. 130(3) : 227-242.

Braun-Blanquet J. \& Bolòs O. de, 1957. Les groupements végétaux du Bassin moyen de l'Èbre et leur dynamisme. Anales de la Estación Experimental de Aula Dei 5(1-4) : 1-266.

Cambecèdes J., Gauquelin T., Roques A., Tébhaut C., Burrus M., Gardes M., Gryta H., Joachim J., Bertaudière V., Rénaux T. \& Largier G., 2005.
Approche intégrée de la conservation des populations pyrénéennes de Genévrier thurifère (Juniperus thurifera L.). Bulletin de la société d'histoire naturelle de Toulouse 141-2: 189-195.

Carreras J., Carrillo E. \& Ferré A., 2000. Mapa de vegetació de Catalunya 1: 50000 : Noarre 150 (34-8) $i$ Tírvia 182 (34-9). Barcelona, Institut Cartogràfic de Catalunya-Institut d'Estudis Catalans.

Coste H. \& Soulié J., 1913. Florule du Val d'Aran. Le Mans, Imprimerie Monnoyer, 32 p.

Davis B.A.S., 1994. Paleolimnology and Holocene environmental change from endorheic lakes in the Ebro Basin, north-east Spain. Ph. D. Thesis. University of Newcastle upon Tyne, 317 p.

Farreny J.E., 1978. Contribució al coneixement de la flora de la Vallferrera. Acta Botanica Barcinonensia $30: 1-118$.

Ferrández J.V., 2003. La sabina albar, Juniperus thurifera L. (Cupressaceae) en el Somontano oriental de Huesca, España. Ecología 17 : 123-130.

Ferrández J.V., 2005. Adiciones y correcciones al catálogo florístico de la comarca del Cinca Medio. Cuadernos del Centro de Estudios de Monzón y Cinca Medio 32 : 43-47.

Font Quer P., 1915. Plantes de Vallferrera. Butlletí de la Institució Catalana d'Història Natural 15 : 51-60.

Gamisans J., 2000. Répartition et écologie du Genévrier thurifère en Corse. In : Gauquelin T., Asmodé J.F. \& Largier G. (éds.), Le Genévrier thurifère (Juniperus thurifera L.) dans le bassin occidental de la Méditerranée : systématique, écologie, dynamique et gestion. Actes du colloque international de Marignac (Haute-Garonne), 26-27 septembre 1997. Paris, ONF, coll. « Les dossiers forestiers » $6: 37-41$.

Garraud L. \& Villaret J.C., 2000. Historique, répartition et données écologiques du Genévrier thurifère dans les Alpes françaises. In : Gauquelin T., Asmodé J.F. \& Largier G. (eds.), Le Genévrier thurifère (Juniperus thurifera L.) dans le bassin occidental de la Méditerranée: systématique, écologie, dynamique et gestion. Actes du colloque international de Marignac (Haute-Garonne), 26-27 septembre 1997. Paris, ONF, coll. «Les dossiers forestiers » $6: 26-36$.

Gauquelin T., Hassani M.I. \& Lebreton P., 1988. Le Genévrier thurifère, Juniperus thurifera L. (Cupressacées) : analyse biométrique et biochimique ; propositions systématiques. Ecologia Mediterranea 14 : 31-42.

Gauquelin T., Bertaudière V., Montes N., Badri W. \& Asmodé J.F., 1999. Endangered stands of thuriferous juniper in the western Mediterranean basin: ecological status, conservation and management. Biodiversity Conserv. 8: 1479-1498.

Gauquelin T., Bertaudière V., Cambecèdes J. \& Largier G., 2003. Le Genévrier thurifère (Juniperus thurifera L.) dans les Pyrénées : état de conservation et perspectives. Acta Botanica Barcinonensia 49 : 8394.

González-Sampériz P., Valero-Garcés B.L., Carrión J.S., Peña-Monné J.L., García-Ruiz J.M. \& Martí-Bono C., 2005. Glacial and Lateglacial vegetation in northeastern Spain: New data and a review. Quatern. Int., 140-141: 4-20.

González-Sampériz P., Valero-Garcés B.L., Moreno A., Jalut G., García-Ruiz J.M., Martí-Bono C., Delgado-Huertas A., Navas A., Otto T. \& Dedoubat J.J., 2006. Climate variability in the Spanish Pyrenees during the last 30,000 yr revealed by the El Portalet sequence. Quaternary Research 66: 38-52. 
Guerby L., 1993. Une nouvelle station à Genévrier thurifère (Juniperus thurifera) dans les Pyrénées : le Quié de Lujat (Ussat-les-Bains, Ariège). Le Monde des plantes $447: 26-27$.

Jalut G., Esteban A., Gauquelin T. Aubert S. Iglesias M. Bouchette A. \& Belet, J.M., 2000. Rôle du genévrier thurifère dans la mise en place de la couverture forestière du sud de l'Europe à la fin du dernier épisode glaciaire. In : Gauquelin T., Asmodé J.F. \& Largier G. (eds.), Le Genévrier thurifère (Juniperus thurifera L.) dans le bassin occidental de la Méditerranée : systématique, écologie, dynamique et gestion. Actes du colloque international de Marignac (Haute-Garonne), 26-27 septembre 1997. Paris, ONF, coll. «Les dossiers forestiers » $6: 160-170$

Jalut G., Esteban A., Bonnet L., Gauquelin T. \& Fontugne M., 2000. Holocene climatic changes in the western Mediterranean, from south-east France to south-east Spain. Palaeogeog., Palaeoclimatol., Palaeoecol. 160: 255-290.

Jiménez J.F., Werner O., Sánchez-Gómez P., Fernández S. \& Guerra J., 2003. Genetic variations and migration pathway of Juniperus thurifera L. (Cupressaceae) in the western Mediterranean region. Israel J. Plant Sci. 51: 11-22.

Jordán de Urriés J., 1954. Memoria adjunta al Mapa Forestal de la provincia de Lérida. Instituto Forestal de Investigaciones y Experiencias. Madrid, 285 p. + mapa en escala $1: 100000$

Labatut G., Lefevre A.C., Montes N. \& Gauquelin T., 2000. Le Genévrier thurifère dans la Haute Ariège. In : Gauquelin T., Asmodé J.F. \& Largier G. (eds.), Le Genévrier thurifère (Juniperus thurifera L.) dans le bassin occidental de la Méditerranée: systématique, écologie, dynamique et gestion. Actes du col loque international de Marignac (Haute-Garonne), 26-27 septembre 1997. Paris, ONF, coll. «Les dossiers forestiers » $6: 42-46$.

Losa M. \& Montserrat P., 1953. Nueva aportación al estudio de la flora de los montes cántabro-leoneses. Anales Inst. Bot. A.J. Cavanilles XI(2) : 385-462.

Marcysiak K., Mazur M., Romo A., Montserrat J.M., Didukh Y., Boratynska K., Jasinska A., Kosinski P. \& Boratynski A., 2007. Numerical taxonomy of Juniperus thurifera, J. excelsa and J. foetidissima (Cupressaceae) based on morphological characters. Bot. J. Linn. Soc. 155: 483-495.

Montserrat P., 1966. La Vegetación de la Cuenca del Ebro. Publicaciones del Centro Pirenaico de Biología Experimental 1(5) : 1-22.
Pèlachs A., 2004. Deu mil anys de geohistòria ambiental al Pirineu central català. Aplicació de tècniques paleogeogràfiques per a l'estudi del territori $i$ el paisatge a la Coma de Burg i a la Vallferrera. $\mathrm{Ph}$. D. Thesis. Universitat Autònoma de Barcelona, Bellaterra, $581 \mathrm{p}$.

Pèlachs A., Soriano J.M. \& Esteban A., 2001. Evolución glaciar y dinámica de la vegetación en el Pirineo central catalán : el complejo glacio-lacustre de Burg (Farrera, Pallars Sobirà, Lleida). Treballs de la Societat Catalana de Geografia 52 : 293-309.

Rivas-Martínez S. et al., 2011. Mapa de series, geoseries y geopermaseries de vegetación de España. Itinera Geobotanica 18-1 : 1-424 y 18-2 : 425-800.

Rodríguez-Ochoa R. \& Pedrol J., 1999. Notes breus (Flora) : Juniperus thurifera L. (Cupressaceae) a la Baixa Ribagorça. Butlletí de la Institució Catalana d'Història Natural 67 : 66.

Romero F., 1983. Flora y vegetación de la cuenca alta del río Luna (León). Monografías ICONA, 29. Madrid, Ministerio de Agricultura, 273 p.

Skuhravá M., 1995. A new gall midge species, Etsuhoa thuriferae sp. n. (Dyptera, Cecidomyidae) from galls on Juniperus thurifera L. (Cupressaceae) in Spain. Zapateri. Revista aragonesa de Entomología 5: 135-146.

Terrab A., Schönswetter P., Talavera S., Vela E. \& Stuessy T.F., 2008. Range-wide phylogeography of Juniperus thurifera L., a presumptive keystone species of western Mediterranean vegetation during cold stages of the Pleistocene. Mol. Phylogenet. Evol. 48: 94-102.

Valero-Garcés B., González-Sampériz P., García-Ruiz J.M., Peña-Monné J.L., Martí-Bono C., Sancho C., Navas, A., Machín J., Delgado-Huertas A., Stevenson A. \& Davis, B. 2004. Paleohydrological fluctuations and steppe vegetation at the last glacial maximum in the central Ebro Valley (NE Spain). Quatern. Int. 122: 43-55.

Villar L. \& Ferrández J.V., 2000. Usos etnobotánicos de la sabina albar y arbustos que le acompañan en Aragón In : Gauquelin T., Asmodé J.F. \& Largier G. (eds.), Le Genévrier thurifère (Juniperus thurifera L.) dans le bassin occidental de la Méditerranée: systématique, écologie, dynamique et gestion. Actes du colloque international de Marignac (HauteGaronne), 26-27 septembre 1997. Paris, ONF, coll. «Les dossiers forestiers » 6: 130-139.

Violant R. 2001. La vida pastoral al Pallars. Ed. Garsineu Tremp, $427 \mathrm{p}$. 